\title{
Role of percutaneous ethanol ablation in malignant liver tumours
}

\author{
Satnam Singh ${ }^{1}$, Jagjeet Singh ${ }^{2}$, Pankaj Dugg*2 ${ }^{*}$ Sanjiv Sharma ${ }^{1}$, Vijay Thakur $^{1}$, Kamaljeet Kaur ${ }^{2}$, Shikha Sood ${ }^{1}$, \\ Moulik Vora ${ }^{1}$, Prabhjyot Singh ${ }^{3}$, Jitender Parmar ${ }^{1}$ \\ ${ }^{1}$ IGMC, Shimla, India \\ ${ }^{2}$ All India Institute of Medical Sciences (AlIMS), India \\ ${ }^{3}$ Govt. Medical College Patiala, India
}

Received: November 11, 2015

Accepted: January 15, 2016

Online Published: February 22, 2016

DOI: $10.5430 / j b g c . v 6 n 1 p 43$

URL: http://dx.doi.org/10.5430/jbgc.v6n1p43

\begin{abstract}
Purpose: To study the effectiveness of image-guided percutaneous ethanol injection (PEI) to ablate malignant hepatic lesions. Methods: It was a hospital based prospective study. Study included 15 patients ( 8 men, 7 women); aged 40-75 years (mean age 58.3 years); involving 4 hepatocellular carcinoma (HCC) (ranging from $2.8 \mathrm{~cm}-8.0 \mathrm{~cm}$ in diameter) and 18 liver metastases (ranging from $0.78 \mathrm{~cm}-4.5 \mathrm{~cm}$ in diameter). Forty eight sessions (18 sessions for 3 HCCs and 30 for 11 liver metastases) using $99.9 \%$ ethanol injection were done under image guidance to treat 21 tumours in 14 patients. Under local anaesthesia, absolute ethanol (99.9\%) was instilled into the tumour via 22 gauge spinal needle. Triple phased Contrast enhanced computed tomography (CECT) was used in all the cases to assess the treatment response.

Results: The 3 patients having solitary HCC tumours measuring $2.8 \mathrm{~cm}, 6.5 \mathrm{~cm}$ and $8 \mathrm{~cm}$ were assigned into complete response, partial response and progressive disease categories respectively after PEI. Treatment response was not assessed in 1 patient. Among 11 liver metastases patients; complete response, partial response, stable disease and progressive disease was assigned in $5(45.5 \%), 1(6.6 \%), 2(18.1 \%)$ and $3(27.2 \%)$ patients respectively.

Conclusions: PEI is a safe and effective technique for treating smaller liver neoplasms and less than 3 lesions for each patient as per BCLC criteria.
\end{abstract}

Key Words: Ablation, Ethanol, Liver, Hepatocellular carcinoma, Metastasis

\section{INTRODUCTION}

Liver tumours including hepatocellular carcinoma (HCC) and metastases are among some of the most common tumours worldwide. Hepatic resection is considered the curative treatment of choice but very few patients are suitable for surgery. Moreover chemotherapy and radiation therapy are ineffective treatment methods especially for HCC. ${ }^{[1]}$ Various percutaneous treatment modalities used for treating liver neoplasms include percutaneous ethanol or acetic acid in- jection, radiofrequency ablation (RFA), microwave ablation, laser photocoagulation, cryotherapy, trans-arterial chemoembolization (TACE), and direct chemotherapeutic agent injection. ${ }^{[2]}$ In percutaneous ethanol injection (PEI) technique, absolute ethanol is directly injected into the lesion through a needle placed into the lesion under image guidance. Ethanol causes dehydration and necrosis of tumour cells, accompanied by small vessel thrombosis, leading to tumour ischemia and destruction. ${ }^{[3,4]}$ It has shown promising results particu-

*Correspondence: Pankaj Dugg; Email: pankajdugg84@gmail.com; Address: All India Institute of Medical Sciences (AIIMS), India. 
larly in HCC lesions less than $3 \mathrm{~cm}$ in diameter. ${ }^{[5]}$ PEI had been less successfully used in treatment of liver secondaries and cholangiocarcinomas. ${ }^{[6-8]}$ Currently, RFA is a first-line treatment of the major local ablative techniques but PEI is preferred in technically difficult masses located in the hepatic dome, in the subcapsular area, and exophytic in position, or are surrounded by large blood vessels. ${ }^{[9]}$

\section{MATERIAls AND METHODS}

A hospital based prospective interventional study was conducted. Informed consent was taken from each patient or their attendants before the study commenced. The study was approved by the institutional review board.

From May 2011 to October 2012, 15 patients (8 men, 7 women; aged 40-75 years; mean age 58.3 years) with 22 liver tumours were recruited for the study. The tumours included 4 HCCs ranging from $2.8 \mathrm{~cm}-8.0 \mathrm{~cm}$ in diameter and 18 liver metastases ranging from $0.78 \mathrm{~cm}-4.5 \mathrm{~cm}$ in diameter and were treated with image guided percutaneous ethanol ablation.

Inclusion criteria - Patients with HCC having single lesion of $3 \mathrm{~cm}-8 \mathrm{~cm}$ in diameter or $\leq 3$ lesions per patient measuring less than $3 \mathrm{~cm}$ in diameter, liver metastasis not suitable for systemic chemotherapy or residual tumour/failure after systemic chemotherapy was having $\leq 3$ lesions of less than $5 \mathrm{~cm}$ in diameter.

Exclusion criteria - Patients with irreversible coagulopathy, prothrombin time ratio $<50 \%$ and platelet count $<50$ $\times 10^{3} / \mathrm{mm}^{3}$, patients with brain metastasis, portal venous thrombosis, massive ascites, and Child-Turcotte-Pugh (CTP) class $\mathrm{C}$ liver cirrhosis.

Laboratory tests including Serum Alpha feto protein (AFP) levels, Serum Albumin, Serum bilirubin, Serum glutamic oxaloacetic transaminase (SGOT), Serum glutamic pyruvic transaminase (SGPT), Prothrombin Time (PT), Prothrombin Time ratio (PTR), International normalized ratio (INR), Platelet count, Renal function test (RFT), Hepatitis B or C antigen were obtained.

All the HCC patients were diagnosed according to European Association for the Study of the Liver (EASL) criteria and all the liver metastases were confirmed with fine needle aspiration cytology (FNAC). In two cases of HCC with cirrhosis diagnosis was made by arterial hypervascularisation on triple phase Contrast enhanced computed tomography (CECT) with washout in Portal venous (PV)/equilibrium phases and elevated Serum AFP levels (> 400 ngm). Arterial enhancement on both triple phase CECT and triple phase Contrast enhanced magnetic resonance imaging (CEMRI) was used in one case of HCC with liver cirrhosis. FNAC was used to confirm one case of HCC with cirrhotic liver.

Twenty-two gauge (G), $7 \mathrm{~cm}$ long spinal needle was used to inject ethanol in all the patients except for $1 \mathrm{HCC}$ patient in which $22 \mathrm{G} 15 \mathrm{~cm}$ long spinal needle was used for 11 sessions. In all the 15 patients, ethanol was instilled under Ultrasonography (USG) guidance. Computed tomography (CT) was additionally used for one treatment session in one patient with HCC. "Toshiba Xario XG" ultrasonography equipment was used for image guidance, assessment of treatment response and follow-up. CT equipment used was a "GE Light Speed VCT Xte" - 64 slice Multidetector computed tomography (MDCT) scanner. Triple phase liver scans were obtained after injecting 80-100 $\mathrm{ml}$ of non-ionic iodinated contrast material at a rate of $3.5 \mathrm{cc} / \mathrm{sec}$ through the antecubital vein by using a power injector (Stellant D; Medrad). MDCT protocol with $.625 \mathrm{~mm}$ collimation, $5 \mathrm{~mm}$ slice thickness, pitch of 1.375 , table speed/gantry rotation of $55 \mathrm{~mm}$, tube current of 100-700 mA with automatic adaptation, $140 \mathrm{kV}$ of tube voltage were used. The arterial phase, Portal venous phase (PVP), equilibrium phase images were obtained at 30-35 sec, 60-70 sec and $180 \mathrm{sec}$ after the initiation of contrast injection. MRI equipment used was a "Seimens Magnatom Avanto1.5 T". Plain axial T1 and T2 weighted images and contrast enhanced triple phase images were obtained after injection of $0.1 \mathrm{mmol} / \mathrm{kg}$ of gadolinium chelate with scan delays similar to as described for multiphase MDCT.

Percutaneous ethanol ablation was performed on admitted patients who were kept fasted for atleast 4-6 hours prior to the procedure. Multi-session conventional technique was used for all subjects comprising of 2-3 sessions per week until the entire tumour was treated. The total volume of ethanol for a given tumour was estimated as $4 / 3 \Pi(r+0.5)^{3}$, whereby $r$ being the tumour radius.

After cleaning and draping the abdomen, $5 \mathrm{ml}-10 \mathrm{ml}$ of $2 \%$ Lignocaine hydrochloride was infilterated subcutaneously at region of interest in all patients. Butorphenol $1 \mathrm{mg}-2 \mathrm{mg}$ intravenously was used in 5 patients for 9 treatment sessions, as they were apprehensive or complained of pain despite adequate local anaesthesia. Under ultrasound guidance, a 22 gauge spinal needle was inserted into the lesion. Absolute ethanol was then administered slowly as $0.2 \mathrm{ml}-0.4 \mathrm{ml}$ boluses every $10 \mathrm{sec}$. Care was taken to inject the deepest portions of the lesion first, then the central and finally the superficial portions. The hyperechoic ethanol diffusion was observed and reposition of needle was done as required. Needle was withdrawn slowly, 30 seconds after injection completion.

Triple phased CECT was used in all the cases to see the 
treatment response. Tumour load was longest diameter of enhancing tumour. Response Evaluation Criteria in Solid Tumours (RECIST) 1.1 criteria was used for tumour response assessment.

\section{RESULTS}

A total of 48 treatment sessions were done to treat 21 tumours in 14 patients. Eighteen sessions of ethanol ablation were performed in $3 \mathrm{HCC}$ tumours and thirty in 11 liver metastases. In one HCC patient, procedure was abandoned. Number of sessions ranged from 1 to 11 per tumour. Maximum of 11 ethanol ablation sessions were done in a case of HCC measuring $6.5 \mathrm{~cm} \times 5.5 \mathrm{~cm}$. The smallest primary liver tumour i.e. $\mathrm{HCC}$ lesion measuring $2.6 \mathrm{~cm}$ required 2 sessions of ethanol ablation. Maximum of 5 ethanol ablation sessions were done in liver metastasis patient having 3 tumours. The smallest liver metastatic lesion measuring $0.78 \mathrm{~cm}$ required only a single PEI session for treatment.

Average volume of ethanol used per tumour was $22.9 \mathrm{cc}$ (range 2.5-110 cc). For HCC it was $65 \mathrm{cc}$ (range 20-110 cc) and $15.9 \mathrm{cc}$ for liver metastasis tumour (range $2.5-45 \mathrm{cc}$ ).

Treatment response was assessed in 3 out of $4 \mathrm{HCC}$ patients (see Table 1). The 3 patients having solitary HCC tumours measuring $2.8 \mathrm{~cm}, 6.5 \mathrm{~cm}$ and $8 \mathrm{~cm}$ were assigned into partial response (see Figure 1), complete response (see Figure 2) and progressive disease categories respectively after PEI (see Table 2). Treatment response was not assessed in 1 patient. Among 11 liver metastasis patients, complete response, partial response, stable disease and progressive disease (see Figure 3) was assigned in 5 (45.5\%), 1 (6.6\%), 2 (18.1\%) and $3(27.2 \%$ ) patients respectively (see Table 3 ).

Table 1. Characteristics of patients with HCC and treatment response

\begin{tabular}{llllll}
\hline $\begin{array}{l}\text { Size of tumour } \\
(\mathbf{c m})\end{array}$ & $\begin{array}{l}\text { S. AFP level } \\
(\mathbf{n g} / \mathbf{d l})\end{array}$ & Associated liver disease & CTP class & $\begin{array}{l}\text { \% reduction in tumour } \\
\text { load }\end{array}$ & $\begin{array}{l}\text { Treatment } \\
\text { response }\end{array}$ \\
\hline 6.5 & 1,069 & Hep.B induced Cirrhosis & A & $57 \%$ & PR \\
8.0 & 1,000 & Hep.B induced Cirrhosis & A & Increased & PD \\
2.6 & 5.5 & Alcohol induced Cirrhosis & B & $100 \%$ & CR \\
\hline
\end{tabular}

Table 2. Tumour characteristics and treatment response in $\mathrm{HCC}$

\begin{tabular}{ll}
\hline No. of patients & $\mathbf{4}$ \\
\hline Tumour number & \\
$\quad$ Solitary & 4 \\
Multiple & 0 \\
Tumour size & \\
$<3 \mathrm{~cm}$ & 1 \\
$3 \mathrm{~cm}-5 \mathrm{~cm}$ & 0 \\
$>5 \mathrm{~cm}$ & 3 \\
Location of tumours & 1 \\
$\quad$ Favourable & 3 \\
unfavourable & 65 \\
Av. Ethanol (ml)/per patient & \\
Treatment response & 1 \\
Complete response & 1 \\
Partial response & 0 \\
Stable disease & 1 \\
Progressive disease & 1 \\
$\quad$ Not assessed &
\end{tabular}

All 4 HCC patients had cirrhosis. Of the $4 \mathrm{HCC}$ cases, two had hepatitis B and 1 had hepatitis C. The fourth HCC patient was a chronic alcoholic. Mild ascites was present in 2 patients with HCC. None had encephalopathy. CTP class A was assigned in three and class B in 1 patient. All (100\%) HCC patients had solitary tumour. Among liver metastasis patients, $6(54.5 \%)$ had solitary and $5(45.4 \%)$ had multiple tumours. Five $(45.4 \%)$ patients had liver metastasis from
Gastrointestinal (GIT) primary, $1(0.9 \%)$ from gallbladder, $2(18.1 \%)$ each from carcinoma cervix and carcinoma lung and $1(0.9 \%)$ from carcinoma ovary (see Table 3$)$. Among liver metastasis tumours of GIT origin, 3 patients had metastatic adenocarcinoma from anorectum, 1 from stomach and 1 from oesophagus. Tumours located close $(\leq 5 \mathrm{~mm})$ to the liver capsule, the gallbladder, the gastrointestinal tract, the inferior vena cava, the hilum, the heart, or the diaphragm were considered as unfavourable sites. Twelve (54.5\%) out of 22 tumours were located at favourable sites and $10(45.4 \%)$ were located at unfavourable sites.

Pain at injection site was the chief complaint in most of the patients. Other complications or side effects were low-grade fever, vomiting, and pneumothorax (see Table 4). There were no major systemic complications.

\section{Discussion}

HCC accounts $4.1 \%$ of all human cancer cases in the world. It is prevalent in Southeast Asia and sub-Saharan Africa, about $70 \%$ and $12 \%$ of all cases was found in Asia and Africa, respectively. Metastatic lesions are most common malignant liver tumours. With the widespread use of AFP, ultrasound, and other diagnostic modalities as screening tools, many small malignant liver tumours are detected before symptoms develop and are treated effectively. ${ }^{[10-12]}$ Partial hepatectomy 
remains the best hope for a cure but is suitable for only $9 \%$ to $27 \%$ of patients. Further resection is often not possible for recurrent tumour because of limited liver reserve. If the tumour is relatively small $(<5 \mathrm{~cm})$, few in number (three or fewer), and confined to the liver, local ablation of the tumour using minimally invasive techniques is a recognized form of treatment. Local ablative therapy has the advantages of preserving the uninvolved liver parenchyma, has no systemic side effects compared to systemic or intra-arterial chemotherapy, and also avoids the morbidity and mortality of major hepatic surgery. Several local ablation therapies, most of which are performed percutaneously under imaging guidance, have been performed as minimally invasive therapy for hepatocellular carcinoma. Among them, PEI has been one of the most widely performed because of its low cost and wide availability.
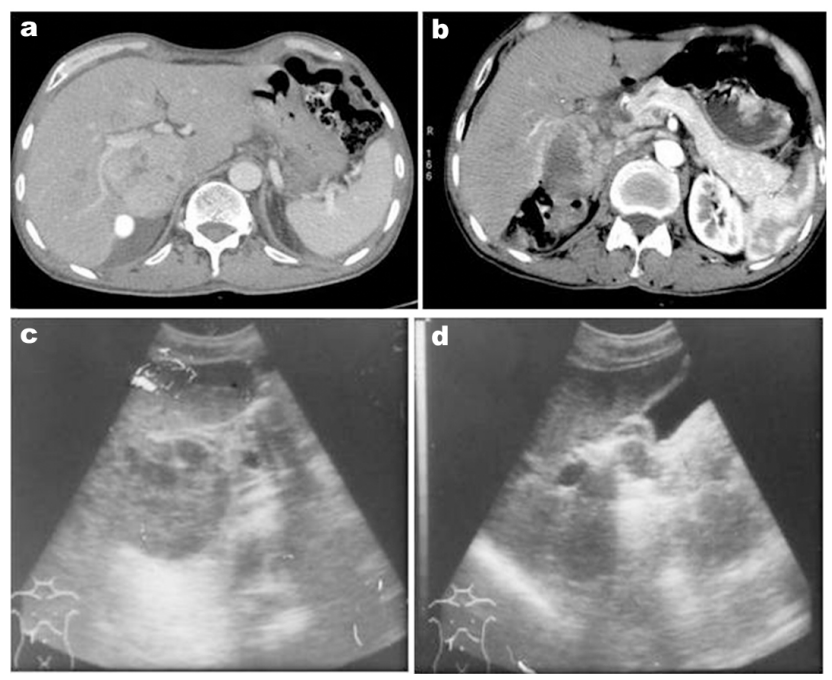

Figure 1. A 48 years old male patient with cirrhosis (CTP class-A) and positive for hepatitis B serology

Pre-ablation late arterial phase image (a) shows heterogeneously enhancing mass of size $6.5 \mathrm{~cm} \times 5.5 \mathrm{~cm}$ in segment I of liver. $S$. AFP was raised $(1,069 \mathrm{ng} / \mathrm{dl})$. Post-ablation arterial phase axial image (b) shows non-enhancement of the necrotic or ablated tumour. In this case necrosis of 57\% was achieved and response was categorised as Partial Response (PR). The medial most part of the tumour could not be injected as it was encased by PV and its right branch. (c) and (d) Ultrasound images taken during ablation showing needle tip and hyperechoic diffusion of ethanol in the tumour.

PEI has shown good results in HCC tumours particularly in $<3 \mathrm{~cm}$ size. Shina et al. ${ }^{[6]}$ has mentioned other indications including liver metastasis, cholangiocarcinoma, peritoneal lymph-node metastasis, adenomatous hyperplastic nodules in the cirrhotic liver, and malignant PV tumour thrombosis in HCC. It is contraindicated in patients with massive ascites, a marked bleeding tendency, or extrahepatic metastasis. Two HCC and four patients with metastasis had mild ascites in our study. Patients with moderate tosevere ascites were not included in the study. Large HCC tumours up to $8.2 \mathrm{~cm}$ and liver metastatic tumours up to $5.8 \mathrm{~cm}$ have been ablated in single session by Ada et al. ${ }^{[11]}$ Giorgio et al. ${ }^{[8]}$ ablated 62 large $(>3.5 \mathrm{~cm})$ liver metastasis with one-shot PEI under general anaesthesia.

Table 3. Tumour characteristics and treatment response in metastasis

\begin{tabular}{ll}
\hline No. of patients & $\mathbf{1 1}$ \\
\hline Tumour number & \\
Solitary & 6 \\
Multiple & 5 \\
Tumour size & \\
$<3 \mathrm{~cm}$ & 13 \\
$3-5 \mathrm{~cm}$ & 5 \\
$>5 \mathrm{~cm}$ & 0 \\
Location of tumours & \\
Favourable & 11 \\
unfavourable & 7 \\
primary tumour & 3 \\
Ca rectum & 1 \\
Ca oesophagus & 1 \\
Ca stomach & 1 \\
Ca ovary & 2 \\
Ca lung & 2 \\
Ca cervix & 1 \\
Ca GB & \\
Treatment response & 5 \\
Complete response & 1 \\
Partial response & 2 \\
Stable disease & 3 \\
Progressive disease & 0 \\
Not assessed & \\
\hline
\end{tabular}

Table 4. Post-ablative complications

\begin{tabular}{lll}
\hline Complication & Episodes & Percentage \\
\hline Pain & 24 & $80 \%$ \\
Fever & 3 & $10 \%$ \\
Vomiting & 2 & $6.7 \%$ \\
Pneumothorax & 1 & $3.3 \%$ \\
Total Episodes & 30 & $100 \%$ \\
\hline
\end{tabular}

We performed forty eight sessions of ethanol ablation using $22 \mathrm{G}, 7 \mathrm{~cm}-9 \mathrm{~cm}$ spinal needle or long spinal needles measuring $15 \mathrm{~cm}$. The main disadvantage with $22 \mathrm{G}$ long spinal needle was that it got clogged repeatedly. So we believe that 18-20 gauge long spinal needle should be used instead of 22 G long spinal needle. Shinna et al. ${ }^{[6]}$ in 1991 introduced a new technique of inserting 2-3 needles into the lesion before start injecting the ethanol to reduce no. of treatment sessions which is the main drawback of conventional technique. 

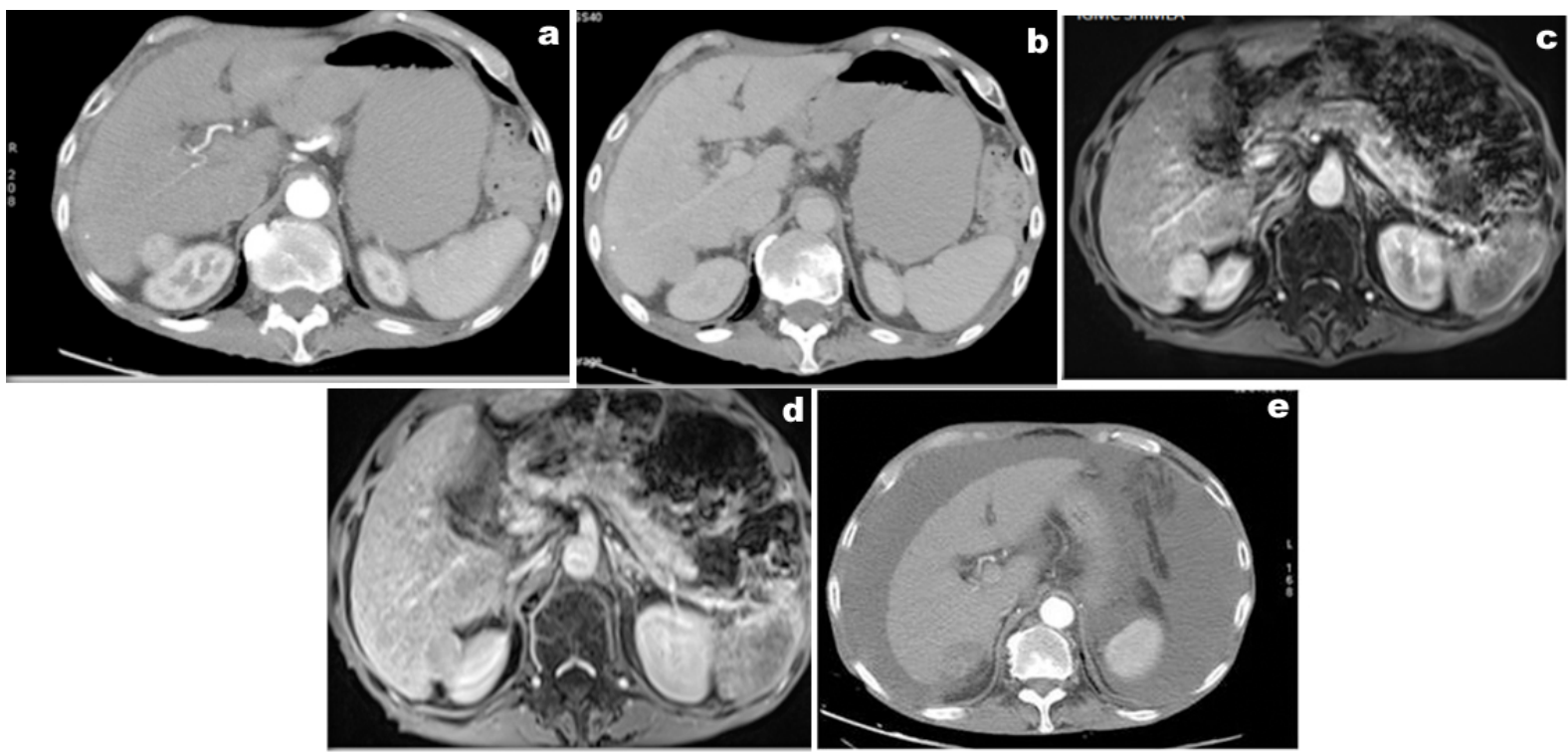

Figure 2. A 72 years old male patient having alcohol induced liver cirrhosis (CTP class B) and oesophagealvarices. S. AFP levels were normal $(5.5 \mathrm{ngm} / \mathrm{dl})$

Axial CECT images (a) arterial phase and (b) equilibrium phase images and axial CEMRI image (c) arterial phase and (d) equilibrium phase images show well defined, rounded-oval, partially exophytic tumour measuring $2.6 \mathrm{~cm} \times 2.4 \mathrm{~cm}$ in segment VI of liver. It show arterial hypervascularisation and wash-out on PVP (not shown) and equilibrium phase images. Capsular enhancement is seen in equilibrium phase MR image (d). Diagnosis of HCC was made on the basis of arterial hypervascularisation and washout in PV or equilibrium phases on two imaging modalities i.e. CECT and CEMRI in the cirrhotic liver. (e) Post ablation follow-up CECT at 4 weeks show complete necrosis of tumour. There is marked ascites which was part of disease process.
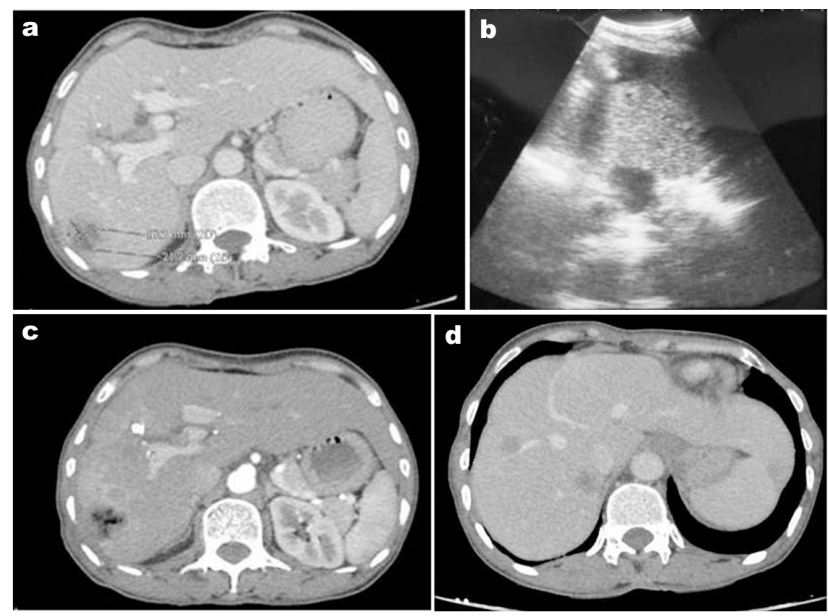

Figure 3. A 48 years old female operated for adenocarcinoma anorectum with single metastatic tumour in segment VII of liver in subcapsular location (unfavourable) Axial CECT image (a) show tumour of size $2.1 \mathrm{~cm} \times 1.8 \mathrm{~cm}$. (b) USG images taken during ethanol ablation. (c) Post-ablation images show complete necrosis of tumour. But the CECT done at an interval of 1 month, $(d)$ shows appearance of multiple new tumours. Patient was assigned into Progressive disease category.

We used ultrasound imaging for guidance during ethanol injection. Fluoroscopy, ultrasound, CT, MRI and CO2enhanced sonography can all be employed for image guidPublished by Sciedu Press ance. ${ }^{[13,15]}$ Ultrasound offered easy manoeuvrability, accurate localisation of tumours and real time monitoring but visibility of tumour and needle was hampered due to echogenicity produced by injected ethanol. So it is advisable to inject the deeper part of the tumour first. CT was additionally used for one treatment session in a patient with HCC without any significant complications.

Triple phased MDCT was used to assess treatment response in all cases. Among the $4 \mathrm{HCC}$ tumours, the smallest tumour measuring $2.8 \mathrm{~cm}$ showed complete necrosis after 2 sessions with $20 \mathrm{cc}$ ethanol injection. Another HCC tumour measuring $6.5 \mathrm{~cm}$ showed partial necrosis (57\%) after PEI. The medial most part of this tumour could not be ablated as it was surrounded by right branch of PV. Another HCC tumour measuring $8 \mathrm{~cm}$ showed no change. Necrosis in $1 \mathrm{HCC}$ tumour measuring $6 \mathrm{~cm}$ was not assessed as the treatment was abandoned due to continuous dribbling of blood through the injected spinal needle and that patient was removed from the study. Kim et al. ${ }^{[14]}$ in 2009 completely ablated 3 HCCs of $<1.5 \mathrm{~cm}$ with PEI instead of using RFA. According to Lencioni et al. ${ }^{[16]}$ complete responses were achieved in $80 \%$ of tumours smaller than $3 \mathrm{~cm}$ in diameter, but only in $50 \%$ of tumours of $3 \mathrm{~cm}-5 \mathrm{~cm}$ in size. The difference in success rate in our study as compared to previous studies could be explained by the fact that our study had larger tumours and 
included tumours that we located at unfavourable sites.

PEI is less successful in ablating liver metastasis. Complete necrosis was seen in $9(50 \%)$ tumours of liver metastasis in our study. Of the 55 liver metastatic tumours ablated with ethanol by Giovannini et al., ${ }^{[7]}$ there was a complete necrosis in 31 cases $(56.3 \%)$ and partial necrosis in 16 cases $(29.1 \%)$ The lesions were unchanged in eight cases (14.1\%). Giorgio et al. ${ }^{[8]}$ treated 14 tumours $(9 \mathrm{HCC}, 4$ liver metastases and 1 lymph node metastasis) with $\mathrm{PEl}$ using Chiba or 22-gauge spinal needle. The success rate for tumours $<3.2 \mathrm{~cm}(11$ cases) was $100 \%$. Tumours $>3.2 \mathrm{~cm}$ ( 3 cases) remained positive for malignant cells. So results of our study is comparable to Giovannini et al. ${ }^{[7]}$ The difference in success rate between Giorgio et al. and our study could be explained by the fact that our study had larger tumours (up to $4.5 \mathrm{~cm}$ liver metastasis).

Most common side effects or complications are pain at injection site and fever. In this study, pain at injection site was the chief complaint in most of the patients. In a study of 14 tumours ablated with PEI by Giorgio et al., ${ }^{[8]}$ patients complained of a local burning pain during and immediately after the injection; this pain was modest in all cases except in one. No systemic complications occurred.

\section{Limitations}

There are few limitations to our study. Firstly, sample size was small in our study. Secondly, there were larger liver tumours in our study as compared to previous studies. Thirdly, most patients have multiple lesions resulting in unfavourable results. Fourthly, we used conventional technique of injection alcohol resulting in more number of sessions needed to inject the required amount of alcohol.

\section{Conclusion}

It was thus concluded that image-guided PEI is a time tested, safe, cheap, and easily available technique for treating liver neoplasms. However, we conclude that PEI should be considered first line treatment option reserved for small hepatocellular tumours, measuring less than $3 \mathrm{~cm}$ in diameter. Its effectiveness in treatment of metastatic liver lesions is variable and should not be recommended for multiple foci of liver lesions ( $>3$ lesions), as this can reduce the treatment response.

\section{REFERENCES}

[1] Gerald D. Minimally Invasive Treatment of Malignant Hepatic Tumors: At the Threshold of a Major Breakthrough. Radiographic. 2000; 20: 9. PMid: 10682768. http://dx.doi.org/10.1148/r adiographics.20.1.g00ja019

[2] Debra AG, Ronald SA. Percutaneous Tumour Ablation for Hepatocellular Carcinoma. AJR. 2011; 197: 789. PMid: 21940565. http://dx.doi.org/10.2214/AJR.11.7656

[3] Schoppmeyer K, Weis S, Mössner J, et al. Percutanous ethanol injection or percutaneous acetic acid injection for early hepatocellular carcinoma. The Cochrane Library. 2010; $1: 1$.

[4] Manish T, Richard HGL, Kiang HT. Interventional Radiology in Treatment of Hepatocellular Carcinoma. SGH proceedings. 2000; 17: 60.

[5] Shinna S, Tateishi R, Yoshida H, et al. Local ablation therapy for HCC. From ethanol injection to radiofrequency ablation. Saudi Med J. 2007; 28: 831-837.

[6] Shiina S, Tagawa K, Unuma T, et al. Percutaneous Ethanol Injection Therapy for the Treatment of Hepatocellular Carcinoma. AJR. 1990 May; 154: 947-951. PMid: 2157329. http://dx.doi.org/10.22 14/ajr.154.5.2157329

[7] Giovannini M, Seitz JF. Ultrasound-Guided Percutaneous Alcohol Injection of Small Liver Metastases. Results in 40 Patients. Cancer. 1994; 73. http://dx.doi.org/10.1002/1097-0142(1994 0115) 73:2<294: :aid-cncr2820730210>3.0.co; $2-j$

[8] Giorgio A, Tarantino L, Mariniello N, et al. Ultrasonography-guided percutaneous ethanol injection in large an/or multiple liver metastasis. Radiol Med. 1998; 96: 238-242.
[9] Jung HK. Is Percutaneous Ethanol Injection Therapy Still Effective for Hepatocellular Carcinoma in the Era of Radiofrequency Ablation? Gut Liver. 2010; 4: 105. PMid: 21103288. http: //dx.doi.org/10.5009/gnl.2010.4.S1.S105

[10] Bruix J, Sherman M, Llovet JM, et al. Clinical Management of Hepatocellular Carcinoma. Conclusions of the Barcelona-2000 EASL Conference. J Hepatol. 2001 Sept; 35: 421-430. http: //dx. doi .o $\mathrm{rg} / 10.1016 / \mathrm{S} 0168-8278$ (01) 00130-1

[11] Ada K, Annat B, Hadar M. Minimally Invasive Techniques in the Treatment of Liver Tumours. IMAJ. 2002; 4: 1106.

[12] O'Toole D, Maire F, Ruszniewski P. Ablative therapies for liver metastases of digestive endocrine tumours. Cancer. 2003; 10: 463. http://dx.doi.org/10.1677/erc.0.0100463

[13] Kazushi N, Katsuaki T, Takayoshi K. Nonresectable Hepatocellular Carcinoma: Improved PEI Therapy Guided by CO2-Enhanced Sonography. AJR. 2001; 177: 789. PMid: 11566674. http://dx.d oi.org/10.2214/ajr.177.4.1770789

[14] Kim SR, Imoto S, Nakajima T, et al. Well-differentiated hepatocellular carcinoma smaller than $15 \mathrm{~mm}$ in diameter totally eradicated with percutaneous ethanol injection instead of radiofrequency ablation. Hepatology Int. 2009; 3: 411-415. PMid: 19669368. http://dx.doi.org/10.1007/s12072-009-9128-z

[15] Achoti, Gregory BB. Management of Hepatic Metastases. Liver Transplantation and Surgery. 1999; 5: 65. http://dx.doi.org/1 $0.1002 / 1 t .500050113$

[16] Lencioni R, Josep M. Percutaneous ethanol injection for hepatocellular carcinoma: Alive or dead. J Hepatol. 2005; 43: 377. PMid: 16005537. http://dx.doi.org/10.1016/j.jhep. 2005 .06 .001 